\title{
Adoption of Electronic Dental Records: Examining the Influence of Practice Characteristics on Adoption in One State
}

\author{
Zain Chauhan $^{1,2}$ Mohammad Samarah ${ }^{3} \quad$ Kim M. Unertl ${ }^{4} \quad$ Martha W. Jones $^{2}$
}

\footnotetext{
${ }^{1}$ Bascom Palmer Eye Institute, University of Miami Miller School of Medicine, University of Miami, Miami, Florida, United States

${ }^{2}$ Center for Medicine, Health, and Society, Vanderbilt University, Nashville, Tennessee, United States

${ }^{3}$ Computer Science and Big Data Analytics, Florida Polytechnic University, Lakeland, Florida, United States

${ }^{4}$ Department of Biomedical Informatics, Vanderbilt University School of Medicine, Vanderbilt University, Nashville, Tennessee, United States
}

Appl Clin Inform 2018;9:635-645.
Address for correspondence Zain Chauhan, MA, Bascom Palmer Eye Institute, University of Miami, 1638 NW 10th Avenue, Suite 706A, McKnight Building, Miami, FL 33136, United States (e-mail: mzc4@miami.edu).

\section{Abstract}

Keywords

- electronic health records and systems

- electronic dental records

- dental clinic

- adoption

- socioorganizational issues
Objective Compared with medicine, less research has focused on adoption rates and factors contributing to the adoption of electronic dental records (EDRs) and certified electronic health records (EHRs) in the field of dentistry. We ran two multivariate models on EDR adoption and certification-capable EHR adoption to determine environmental and organizational factors associated with adoption.

Methods We conducted telephone survey of a 10-item questionnaire using disproportionate stratified sampling procedure of 149 dental clinics in Tennessee in 2017 measuring adoption of dental information technology (IT) (EDRs and certificationcapable EHRs) and practice characteristics, including region, rurality, specialty, and practice size. We used binomial logistic regression models to determine associations of adoption with predictor variables.

Results A total of $77 \%$ of surveyed dental clinics in Tennessee had adopted some type of EDR system. When the definitions of certification capable were applied, the adoption rates in dental clinics dropped to $58 \%$. A binomial logistic regression model for the effects of rurality, specialization, and practice size on the likelihood that a clinic would adopt an EHR product was statistically significant (chi-square $(3)=12.41, p=0.0061$ ). Of the three predictor variables, specialization and practice size were significant: Odds of adopting an EHR is $67 \%$ lower for specialists than for general dentists; and clinics with two or more practicing dentists were associated with a much greater likelihood of adopting an EHRcapable system (adjusted odds ratio $=3.09, p=0.009$ ).

Conclusion Findings from this study indicate moderate to high levels of overall dental IT adoption. However, adoption rates in dental clinics do remain lower than those observed in office-based physician practices in medicine. Specialization and practice size were significant predictors of EHR-capable system adoption. Efforts to increase EHR adoption in dentistry should be mindful of potential disparities in smaller practices and between dental specialties and generalists. received

May 2, 2018

accepted after revision June 25, 2018 (c) 2018 Georg Thieme Verlag KG Stuttgart · New York
DOI https://doi.org/

10.1055/s-0038-1667331. ISSN 1869-0327. 


\section{Background and Significance}

Oral health care is an integral component to the overall health of an individual. ${ }^{1}$ However, dental and medical fields have often operated in separate domains-divided by policy, insurance, education, and professionalization. ${ }^{2}$ Recently, movements to improve interprofessional collaboration ${ }^{3}$ have suggested the potential value of exchanging patient data in bridging the dental-medical divide. ${ }^{3,4}$ A study in 2017 found that medical care providers reported substantial value in having access to a patient's oral health data. ${ }^{5}$ The appropriate exchange of patient data, such as medical history, dental history, laboratory reports, and prescribed medications, between dental and medical clinics has the potential to breakdown structural barriers that obstruct medical and dental communication, allow for better cooperation between medical and dental clinics, and provide more reliable data on a patient's overall health. ${ }^{3,6,7}$

One of the essential first steps to an integrated medicaldental patient data environment is the development, adoption, and widespread use of certified interoperable electronic health record (EHR) systems in both the dental and medical fields. Certified EHRs are software systems that have been tested and certified by the Office of the National Coordinator for Health Information Technology (ONC), and, thereby, meet certain standards and functions required for Meaningful Use and interoperability attestation. Many studies have analyzed the adoption and factors that contribute to the adoption of EHRs in medicine. ${ }^{8-14}$ The ONC has documented increased adoption of EHRs across health care contexts, ${ }^{15}$ due in part to the Health Information Technology for Economic and Clinical Health (HITECH) Act and Meaningful Use/Promoting Interoperability mandate. In dentistry, on the other hand, there have been fewer studies analyzing the adoption of dental information technology (IT), such as electronic dental records (EDRs), ${ }^{7,16-19}$ with significantly less research focused on examining factors associated with adoption. The consensus from most dental researchers and practitioners is that the adoption of IT software in dentistry has been slower than in the medical field. ${ }^{20}$ The most recent findings from a national study in the United States in 2012 found an overall self-reported EDR adoption rate of 52\%. ${ }^{19}$ For comparison, the national-level adoption of "any EHR" in an office-based physician practice in the same year was $71.8 \% .^{15}$

As the health care field becomes more integrated, and health care IT becomes more interoperable, it has been predicted that the environment (e.g., location) and organizational structure (e.g., practice size) will play an ever-increasing role in influencing and modulating adoption decisions. ${ }^{20}$ In this study, we have recorded the adoption rates of dental EDRs in dental clinics across the state of Tennessee and examined environmental and organizational factors associated with adoption. To take into account the broad range of dental IT systems that fall into the category of an EDR, we examined the adoption of basic EDR systems and those with capabilities of meeting minimum criteria and clinical quality measures for EHR certification.

\section{Objectives}

The overarching research question driving this study is: What are the rates and practice characteristics that are associated with the adoption of EDRs? We aimed to measure the adoption rates of EDRs and certification-capable EHRs in dental clinics in Tennessee through a telephone survey. We ran two multivariate models on EDR adoption and certification-capable EHR adoption to determine environmental and organizational factors associated with adoption. We examined rates across practice characteristics, such as location (i.e., oral health region and rurality), size of the practice, and specialty.

\section{Materials and Methods}

\section{Sampling Frame}

Statewide representative dental clinic telephone survey data were collected in 2017 in Tennessee. The target population was all dental clinic offices, which included general dental offices and specialty practices. The sampling frame was obtained from the Tennessee Department of Health (TDH), based on publicly available licensure reports of all dentists who are licensed to practice in Tennessee. ${ }^{21}$ These reports include information such as race, gender, date of graduation, clinic location, clinic contact information, and limited specialty information. Because the unit of analysis in this study is the dental clinic (and not the individual dentist), this data set was normalized to uniquely represent each office. The normalization process led to the size of the data set decreasing from 5,770 individual dental licensing numbers in the original data set to 2,066 dental clinics in the normalized data set.

\section{Survey Design}

The survey included an opening script, a consent script, and a 10-item questionnaire (see - Appendix A for survey overview). The survey was developed with the objectives of the study in mind with input from dentists, dental assistants, and survey specialists at the Vanderbilt University Department of Health Policy and Center for Medicine, Health, and Society. The content validity and stability of the survey was determined by pretesting on several dental clinics. Feedback from dentists and dental staff guided the development of the finalized instrument. The survey was estimated to take around $3 \mathrm{~min}-$ utes to complete. The dental office was selected as the primary unit of analysis for several reasons. Prior to designing the survey, a direct observational study was conducted by the authors to assess clinic workflow in rural and urban dental clinics. One of the findings showed that dental staff, as opposed to the dentist, were the primary users of dental IT, from booking to inputting treatment and medication data. A dental workflow study in 2016 showed similar findings. ${ }^{22}$ Interviews from several members of the dental staff team indicated that staff were well-versed in the type of dental IT product used in the clinic. Furthermore, in both clinics observed, the staff were integral in the decision-making process of the type of IT system the dentist purchased. This finding falls in line with studies that have indicated that health IT adoption is an office-level decision instead of an individual decision. ${ }^{12,23}$ 


\section{Survey Measures}

Independent Variables: Oral Health Region, Rurality, Practice Size, and Specialty

The regional location was determined based upon the county where the clinic resided. The region was defined using the seven TDH oral health regions, all containing a regional office and a central public health dental clinic. ${ }^{24}$ Each of the counties represented was then giving a numerical value using the Index of Relative Rurality (IRR). The IRR, which was introduced by Dr. Brigitte Waldorf ${ }^{25}$ at Purdue University, provides a continuous measure of the relative rurality of a county based on four dimensions: population size, density, percentage of urban residents, and distance to the closest metropolitan area. The rurality measure varies from 0 to 1 , with 0 being the most urban and 1 being the most rural. The exact value for the rurality of each of Tennessee's 95 counties using the IRR has been previously calculated by the Tennessee Advisory Commission on Intergovernmental Relations and published in a 2016 report. $^{26}$-Fig. 1 details the geographic distribution of the IRR in the sampling frame. For comparative purposes, we also examined adoption across the U.S. Office of Management and Budget (OMB) county-level dichotomous metropolitan/ nonmetropolitan categories. ${ }^{26}$

The practice size was estimated by the number of dentists practicing in the clinic, and was categorized into 1 or $\geq 2$ and was assessed by the question, "How many dentists work at this practice?" The number of practitioners working in a clinic is often used as a substitute for practice size. ${ }^{12,27}$ Specialty clinics were defined based on approved definitions by the Council on Dental Education and Licensure (e.g., oral surgery, orthodontist, periodontist, etc.). ${ }^{28}$ Each clinic surveyed was asked if the clinic was a general or specialty practice. A follow-up question determined what type of specialty, or specialties, were practiced.

\section{Dependent Variables: EDR and EHR Adoption}

Most health IT record-keeping systems in the medical and dental fields fall into the categories of EHRs, electronic medical records (EMRs), and, in the case of dentistry, EDRs. Informally, these terms are often used interchangeably and broadly describe a computer system used for tracking a patient's clinical data electronically. The ONC, however, reserves the term EHR for software systems that are capable of sharing data between multiple health care organizations. ${ }^{29}$ EHR products that are certified must be capable of meeting a set of functions that is defined in the Certified Health IT Product List. ${ }^{30}$ It should be noted that system functionality, design, and architecture still differ between certified EHR products. ${ }^{31}$ The data stored within noncertified EMRs are usually not shared so readily. EDRs that are not certified through the ONC can be considered comparable in functionality to EMRs. However, due to the lack of consistency in clinical terminology, data standards, and controlled vocabularies between various EDR systems, it is difficult to make direct comparisons. ${ }^{32}$

To take into account the wide range of dental IT products used among the surveyed clinics, products were categorized into EDRs and certification-capable EHRs. EDRs were defined as any dental IT product that is capable of storing patient information, from electronic practice management systems with clinical tools to fully certified EHRs. Therefore, we considered all certified EHRs to also be EDRs, but not all EDRs qualified as a certification-capable EHR. For our study, to qualify as a certification-capable EHR, the product must have a version that meets certification requirements as defined in the Certified Health IT Product List generated by the ONC. ${ }^{33}$ For example, suppose that three pediatric dental clinics were sampled. The first clinic reported the use of DOX | Pedo, another the use of Cloud9Pedo, and the last was predominantly paper-based. The first clinic is using a software suite capable of being upgraded to a full EHR (DOX | Pedo Version 2.0), while the latter two are not. ${ }^{34}$ Based on our definitions, we classify DOX |Pedo as an EDR and certification-capable EHR, Cloud9Pedo as an EDR only, and paper-based as neither.

The adoption of EDRs was measured based on the survey question, "Does your practice currently use an EDR keeping system of some kind?" The reason that this language was used, specifically "EDR" and "of some kind," was an attempt to encompass the wide range of IT systems used in dentistry that may fall in the category of an EDR. ${ }^{7}$ The specific type of health IT system used was then determined by the question, "What is the name of the EDR system you are using?" Having the specific name of the product used in the clinic allows for determining if the system has been certified by the Centers for Medicare \& Medicaid Services and ONC. ${ }^{34}$ If the participant said they do not use an EDR "of some kind," the interviewer clarified the response by asking if the clinic is predominantly paper-based.

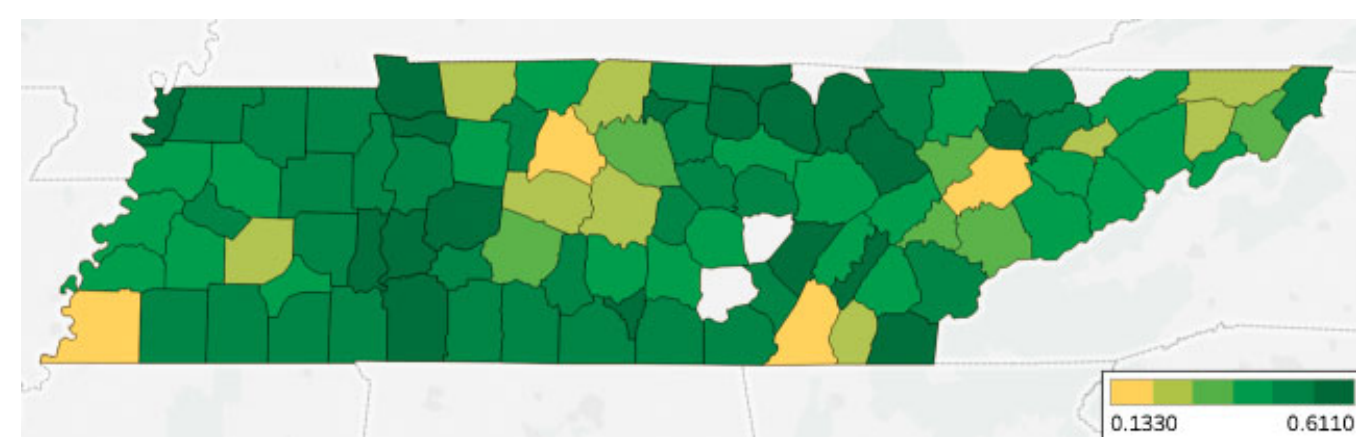

Fig. 1 Geographical distribution of Index of Relative Rurality by county in the sampling frame. Lower scores represent more urban on the IRR scale. The lowest score was 0.1330 (Shelby County), while the highest score was 0.611 (Perry County). Four counties were not represented. 


\section{Analyses}

\section{Categorization and Sampling}

For sampling and analytic purposes, the IRR for each county was stratified into four groups: urban, low rurality, medium rurality, and high rurality, with urban and high rurality representing the least and most rural, respectively. To do this while avoiding arbitrarily categorizing the IRR, the $K$ means clustering method was used on the cleaned data set. $\mathrm{K}$-means is a technique that is designed to group similar observations in a data set, such that observations in the same group are as similar to each other as possible and observations in different groups are as different from each other as possible. ${ }^{35}$ The final clustering is dependent upon both the initial centroid position and the initial $K$-value that is picked. The initial centroid position was determined by randomly assigning a value and then, through a dispersed method, selecting the farthest available point for the next centroid. To avoid bias in selecting a $K$-value, a scree plot and a search for a kink in the curve generated from the within sum of squares (WSS) for numerous cluster solutions was used. After 20 cluster solutions ( $K$-values of $1-20$ ) were tested with random starting points, a kink was found in the WSS curve at a $K$ value of 4 . The final categorization generated from the $K$ means cluster and descriptive statistics for the intervals are detailed in - Table 1.

Due to the relatively low number of clinics in the medium and high rurality clusters, a disproportionate stratified sampling procedure was used to adequately compare differences in adoption between rural and urban areas. The stratum used was rurality category. Initially, simple random sampling was conducted in each rurality category until a total of 25 survey responses in each category were recorded with a total of 100 completed surveys. A review of the data showed an insufficient number of specialty clinics were sampled. Simple random sampling was conducted on the population until a total of 50 additional survey responses were recorded. Of the 50 survey responses, one was determined to be ineligible as the clinic was affiliated with a university that was using multiple IT systems.

\section{Weighted Ratios}

The process of initially sampling an equal number of dental clinics in each rurality category led to $4.74 \%$ of urban offices, $8.4 \%$ of low rurality offices, $9.19 \%$ of medium rurality offices, and $12 \%$ of high rurality offices getting selected. To increase precision, poststratification weight adjustments were made based on sampling frame characteristics in the cleaned data set for analysis of rural-urban differences. Furthermore, since regional information was available for all observations in the sampling frame, it was selected as another characteristic to poststratify and was used in bivariate analysis.

\section{Association of Dental IT Adoption and Independent Variables}

Prior to running multivariate analyses, we examined the relationships between explanatory variables. As expected, collinearity was observed between oral health region and rurality. Furthermore, due to an inadequate sample size for oral health region as established according to Cochran ${ }^{36}$ for chi-square tests, oral health region was dropped from multivariate procedures. Instead, a Fisher's exact test adjusted for poststratification weights was used to determine relationships between dental IT adoption and oral health region. Two logistic regression models were conducted to assess factors that were associated with two outcomes of interest: EDR and EHR adoption. The predictor variables for both regression models were the same and included rurality, practice size, and specialization. Previous studies conducted in the medical field have indicated that these factors may be associated with adoption. ${ }^{14,37,38}$ IRR rurality designations were used in multivariate analysis. All analyses were performed using Stata Release 14.2 (StataCorp LP).

\section{Results}

\section{Sample Distribution and Descriptive Statistics}

Overall, 149 successful surveys were recorded. There was a response rate of $36.3 \%$ ( 149 surveys/410 telephone calls). When comparing nonrespondents to respondents, we found that nonrespondents were more likely to be from the urban and low rurality categories $(p=0.012)$. Descriptive statistics for the study, reported in - Table 2, show that the sample consisted of 45 urban clinics, 52 low rurality clinics, 25 medium rurality clinics, and 27 high rurality clinics. All of the seven TDH regions were represented in the sample, with the Mid-Cumberland region (21\%) being the largest surveyed and Upper Cumberland (8\%) being the lowest. Most of the clinics surveyed (56\%) identified as being general practice. - Fig. 2 details the spatial distribution of the prevalence of general practice clinics and

Table 1 Descriptive statistics for IRR categories for 2,066 dental offices in cleaned data set

\begin{tabular}{|l|l|l|l|l|l|l|}
\hline Rurality category & Centroid $^{\mathbf{a}}$ & Lower bound & Upper bound & $\begin{array}{l}\text { Frequency } \\
\text { (no. of offices) }\end{array}$ & Relative frequency & $\begin{array}{l}\text { Frequency } \\
\text { (no. of counties) }\end{array}$ \\
\hline Urban & 0.152 & 0.133 & 0.236 & 950 & 0.460 & 4 \\
\hline Low & 0.270 & 0.237 & 0.344 & 619 & 0.300 & 12 \\
\hline Medium & 0.392 & 0.345 & 0.446 & 272 & 0.132 & 21 \\
\hline High & 0.491 & 0.447 & 0.611 & 225 & 0.109 & 53 \\
\hline
\end{tabular}

Abbreviation: IRR, Index of Relative Rurality.

${ }^{a}$ Data point at the center of the cluster.

b Lower value indicates more urban on the IRR scale. 
Table 2 Descriptive statistics of study sample and sampling frame

\begin{tabular}{|c|c|c|c|c|}
\hline Variable & $\begin{array}{l}\text { Number } \\
\text { of cases }\end{array}$ & $\begin{array}{l}\text { Percentage } \\
\text { of sample }\end{array}$ & $\begin{array}{l}\text { Number of cases } \\
\text { in sampling frame }\end{array}$ & $\begin{array}{l}\text { Percentage of } \\
\text { sampling frame }\end{array}$ \\
\hline \multicolumn{5}{|l|}{ Rurality } \\
\hline Urban & 45 & 30 & 950 & 46 \\
\hline Low & 52 & 35 & 619 & 30 \\
\hline Medium & 25 & 17 & 272 & 13 \\
\hline High & 27 & 18 & 225 & 11 \\
\hline \multicolumn{5}{|l|}{ Clinic type } \\
\hline General & 83 & 56 & & \\
\hline Specialty & 66 & 44 & & \\
\hline \multicolumn{5}{|l|}{ Region } \\
\hline East & 22 & 15 & 361 & 18 \\
\hline Mid-Cumberland & 31 & 21 & 670 & 32 \\
\hline Northeast & 20 & 13 & 144 & 7 \\
\hline South Central & 13 & 9 & 92 & 4 \\
\hline Southeast & 22 & 15 & 223 & 11 \\
\hline Upper Cumberland & 12 & 8 & 72 & 4 \\
\hline West & 29 & 19 & 504 & 24 \\
\hline \multicolumn{5}{|l|}{ Size } \\
\hline 1 Dentist & 100 & 67 & & \\
\hline$\geq 2$ Dentists & 49 & 33 & & \\
\hline Overall sample & 149 & 100 & 2,066 & 100 \\
\hline
\end{tabular}

Note: "Number of cases" values represent the frequency of dental offices per category.

specialists within the 46 representative counties sampled; colors represent the sum of value labels in the county (general practice $=0$; specialist $=1$ ), with lighter colors representing a greater number of general practice clinics. Most practices consisted of one practicing dentist (67\%).

\section{Factors Associated with Health IT Adoption}

All clinics successfully surveyed had either adopted a health IT product or predominantly used a paper-based recordkeeping system. A majority of clinics (77\%) had adopted some kind of EDR product, while $58 \%$ had adopted a certificationcapable EHR. The regions with the highest adoption of an EDR were the neighboring regions of East Tennessee and Southeast Tennessee (86\%). The region with the lowest overall adoption was West Tennessee (62\%). Despite there being a cluster of high adoption in the East, Southeast, Upper Cumberland, and Mid-Cumberland regions, there was no overall evidence of a relationship between oral health region and $\operatorname{EDR}(p=0.118)$ or certified EHR adoption $(p=0.954)$.

Seventy-eight percent of the urban dental clinics surveyed had adopted a health IT product of some kind compared with $63 \%$ in the high rurality category. After it was determined whether the EDR used by the clinics qualified as being a certification-capable EHR, the adoption rate in the urban

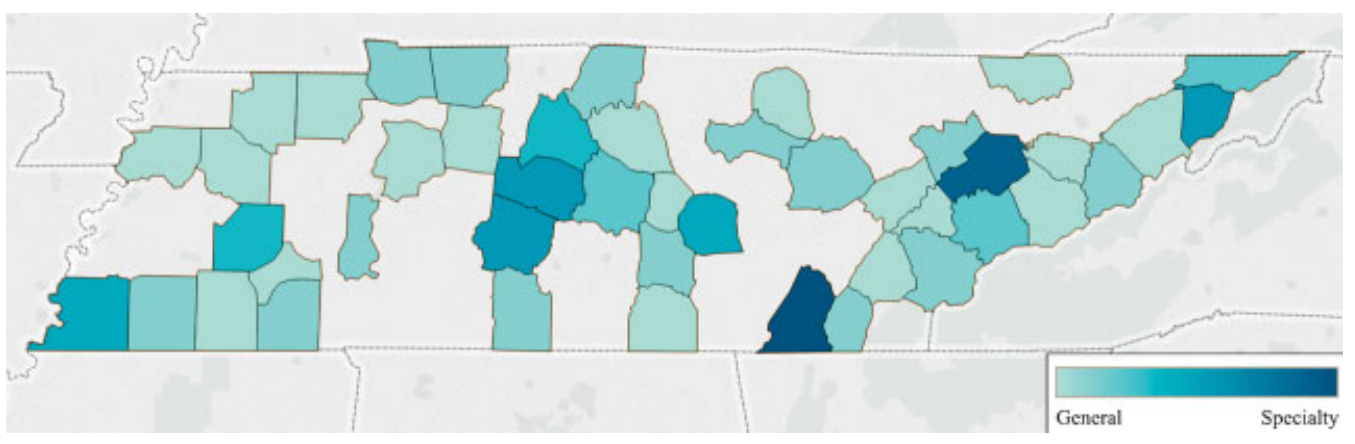

Fig. 2 Prevalence of sampled general and specialty clinics within counties. Colors represent the sum of value labels of general and specialty clinics (general $=0$; specialty $=1$ ). Lighter colors indicate a greater number of general practice clinics. 
category dropped to approximately $51 \%$, with adoption of a certification-capable EHR in high rurality areas at $52 \%$. The adoption of EHRs in low rurality and medium rurality counties was found to be 62 and 67\%, respectfully. There was no association found between adoption of an $\operatorname{EDR}(p=0.380)$ or certified EHR $(p=0.534)$ and rurality as defined by the IRR. After metropolitan/nonmetropolitan category definitions were applied, we found that $80 \%$ of clinics in metropolitan counties had adopted an EDR compared with 69\% in nonmetropolitan counties. For EHR-capable systems, adoption dropped to $59 \%$ in metropolitan counties and 53\% in nonmetropolitan counties. There was also no association found between adoption and metropolitan/nonmetropolitan categories as defined by the OMB for EDRs $(p=0.165)$ and certification-capable EHRs $(p=0.548)$ (see - Table 3$)$.

In general practice clinics, $78 \%$ had adopted an EDR of some kind compared with $76 \%$ in the specialty category. For the adoption of a certification-capable EHR, 69\% of generalists had adopted compared with $44 \%$ of specialists, a significant difference in proportions of 0.25 , 95\% confidence interval $(0.09,0.41), p=0.003$. The relative frequency of EDR adoption in clinics consisting of one practicing dentist was $74 \%$ compared with $84 \%$ for clinics with two or more dentists. This difference was more notable when the EHR definitions were applied, 52 and $70 \%$ respectively (see - Fig. 3 ).

A binomial logistic regression model for the effects of rurality (IRR), specialization, and practice size on the likelihood that a clinic would adopt an EHR-capable product was statistically significant (chi-square $(3)=12.41, p=0.0061$ ). Of the three predictor variables, specialization and practice size were significant (see -Table 4). Specialists were less likely to adopt compared with general practice clinics. The odds of adopting an EHR were 67\% lower for specialists than

Table 3 EDR and certification-capable EHR adoption by region, rurality, clinic type, and clinic size

\begin{tabular}{|c|c|c|c|c|c|c|}
\hline \multirow[t]{3}{*}{ Variable } & \multicolumn{3}{|c|}{ Any EDR adoption (\%) } & \multicolumn{3}{|c|}{ EHR capable adoption $(\%)^{b}$} \\
\hline & Yes & No & \multirow[t]{2}{*}{$p$-Values $^{a}$} & Yes & No & \multirow[t]{2}{*}{$p$-Values $^{\mathrm{a}}$} \\
\hline & $(N=115)$ & $(N=34)$ & & $(N=82)$ & $(N=60)$ & \\
\hline \multicolumn{7}{|l|}{ Region } \\
\hline East & 86 & 14 & \multirow[t]{7}{*}{0.118} & 62 & 38 & \multirow[t]{7}{*}{0.954} \\
\hline Mid-Cumberland & 84 & 16 & & 57 & 43 & \\
\hline Northeast & 70 & 30 & & 60 & 40 & \\
\hline South Central & 69 & 31 & & 67 & 33 & \\
\hline Southeast & 86 & 14 & & 48 & 52 & \\
\hline Upper Cumberland & 83 & 17 & & 64 & 36 & \\
\hline West & 62 & 38 & & 55 & 45 & \\
\hline \multicolumn{7}{|l|}{ Rurality (IRR) } \\
\hline Urban & 78 & 22 & \multirow[t]{4}{*}{0.380} & 51 & 49 & \multirow[t]{4}{*}{0.534} \\
\hline Low & 85 & 15 & & 62 & 38 & \\
\hline Medium & 76 & 24 & & 67 & 33 & \\
\hline High & 63 & 37 & & 52 & 48 & \\
\hline \multicolumn{7}{|l|}{ Metro/Nonmetro } \\
\hline Metropolitan & 80 & 20 & \multirow[t]{2}{*}{0.165} & 59 & 41 & \multirow[t]{2}{*}{0.548} \\
\hline Nonmetropolitan & 69 & 31 & & 53 & 47 & \\
\hline \multicolumn{7}{|l|}{ Clinic type } \\
\hline General & 78 & 22 & \multirow[t]{2}{*}{0.712} & 69 & 31 & \multirow[t]{2}{*}{$0.003^{c}$} \\
\hline Specialty & 76 & 24 & & 44 & 56 & \\
\hline \multicolumn{7}{|l|}{ Size } \\
\hline 1 Dentist & 74 & 26 & \multirow[t]{2}{*}{0.186} & 52 & 48 & \multirow[t]{2}{*}{$0.034^{c}$} \\
\hline$\geq 2$ Dentists & 84 & 16 & & 70 & 30 & \\
\hline Overall adoption & 77 & 23 & & 58 & 42 & \\
\hline
\end{tabular}

Abbreviation: EDR, electronic dental record; EHR, electronic health record; IRR, Index of Relative Rurality; ONC, Office of the National Coordinator for Health Information Technology.

Note: Numbers represent percentage adoption in each category.

${ }^{a} p$-Values for chi-square tests and design-adjusted Fischer's exact test for oral health region.

${ }^{\mathrm{b}}$ Certified EHRs were determined through ONC certification report.

Indicates statistically significant result 


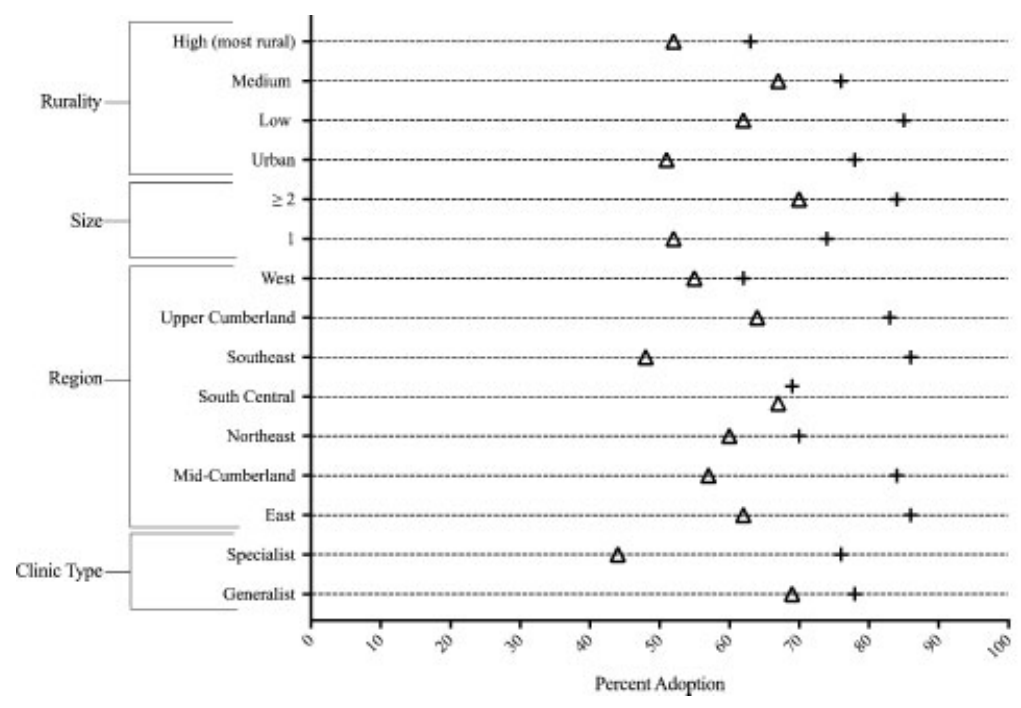

+ EDR.Adoption

$\Delta$ EHR Capable Adoption

Fig. 3 Prevalence of electronic dental record (EDR) and electronic health record (EHR) capable adoption among Tennessee dental clinics by practice size, oral health region, rurality, and clinic type.

for general dentists. Clinics with two or more practicing dentists were associated with a much greater likelihood of adopting an EHR-capable system (adjusted odds ratio $=3.09, p=0.009$ ). The regression model examining EDR adoption was not significant for any of the predictor variables (see - Table 4).

\section{Discussion}

Increasing the adoption of interoperable EHRs has been one of the central goals of the U.S. health care system with the advent of the HITECH Act, which provided approximately 27 billion dollars of federal stimulus funds to speed up the adoption of EHRs. ${ }^{39}$ Data indicate that adoption rates in the medical field increased substantially with the implementation of the HITECH Act. From 2008 to 2015, office-based physician adoption of EHRs rose from 42 to $87 \%$ with an overall use of certified EHRs at $77.9 \%$ as of $2015 .{ }^{40}$

The impact of the HITECH incentive structure on dental EDR and EHR adoption is not well-known due, in part, to the lack of data. ${ }^{20}$ However, because most dentists do not qualify for the federal incentive program under the HITECH Act ${ }^{41}$ as well as the high upfront cost of purchasing an EDR/EHR system, ${ }^{42}$ we hypothesized that dental adoption lags behind medicine. Findings from this study indicate moderate to high levels of overall dental IT adoption. However, adoption rates in dental clinics do remain lower than the rates observed in the medical field. In $2015,87.4 \%$ of office-based physicians in Tennessee had adopted any EHR product ${ }^{15}$ compared with 77\% EDR adoption in dental clinics in 2017 in our study. When the definitions of certification capable were applied, the adoption rates in dental clinics dropped to $58 \%$.

Table 4 Logistic regression of EDR and certification-capable EHR adoption (2017)

\begin{tabular}{|c|c|c|c|c|c|c|}
\hline \multirow[t]{2}{*}{ Variable } & \multicolumn{3}{|c|}{ EDR adoption } & \multicolumn{3}{|c|}{ EHR capable adoption } \\
\hline & Odds ratio & $95 \% \mathrm{Cl}$ & $p$-Values & Odds ratio & $95 \% \mathrm{Cl}$ & $p$-Values \\
\hline \multicolumn{7}{|l|}{ Rurality } \\
\hline \multicolumn{7}{|l|}{$(\text { Urban })^{\mathrm{a}}$} \\
\hline Low & 1.617 & $0.548-4.778$ & 0.384 & 1.541 & $0.636-3.737$ & 0.338 \\
\hline Medium & 0.883 & $0.259-3.013$ & 0.842 & 1.522 & $0.477-4.855$ & 0.478 \\
\hline High & 0.475 & $0.158-1.433$ & 0.186 & 0.871 & -0.260 to 0.798 & 0.798 \\
\hline Clinic type (generalist) & 0.959 & $0.398-2.313$ & 0.926 & 0.332 & $0.151-0.733$ & $0.006^{\mathrm{b}}$ \\
\hline Size (1 dentist) & 2.336 & $0.914-5.969$ & 0.076 & 3.09 & $1.319-7.256$ & $0.009^{\mathrm{b}}$ \\
\hline Intercept & 4.139 & $1.176-14.573$ & $0.027^{b}$ & 1.403 & $0.504-3.903$ & 0.517 \\
\hline Pseudo- $R^{2}$ & 0.027 & & & 0.089 & & \\
\hline Number of obs. & 149 & & & 142 & & \\
\hline
\end{tabular}

Abbreviations: $\mathrm{Cl}$, confidence interval; EDR, electronic dental record; EHR, electronic health record.

Note: Index of Relative Rurality (IRR) categories were used in the logistic regression model.

aReference categories are in parentheses.

Indicates statistically significant result. 
Based upon previously published literature, we hypothesized that there would be relationships between adoption of dental IT and region, rurality, practice size, and clinic type (general or specialty). ${ }^{37,43-49}$ Previous work in the medical field has operationalized regional differences in EHR adoption as the percentage of physicians in the same hospital referral region (HRR) that adopted an EHR. ${ }^{27}$ HRR represents the potential to share data between medical care providers and serves as the geographic proxy for examining regional IT adoption..$^{50}$ However, because there is no reason to believe that referral patterns for dental care in a region overlap with those observed in medical care, TDH oral health regions were used instead. We have shown in this study that dental clinics in different regions had comparable rates of adoption, limiting the influence of a regional factor on driving adoption decisions. This finding falls in line with studies detailing the prevalence of small, individualistic, and disaggregated solo practices in dentistry. ${ }^{44,51}$

Medium and low rurality counties had the highest levels of adoption for certification-capable EHRs. For EDR adoption, we found that the most rural counties on the IRR scale had the lowest rates. However, we found no statistically significant differences between adoption rates in varying rurality categories for both dental IT categories. This finding is similar to several national-level studies that have examined adoption of EMRs in the medical field. ${ }^{12,38}$ However, it is difficult to make direct comparisons with these studies due to different methodological approaches. For example, other studies examining rural-urban differences did not use the IRR to define rural and urban areas. The way rural and urban areas are defined and categorized may influence outcomes. It was expected that rurality may overlap with the other variables examined in this study, namely, practice size and practice region. Despite there being a relationship between region and rurality, there was no association between practice size and rurality. Large offices were present in both urban and high rurality areas in near equal proportions. Because most studies in the medical field have used more traditional metro/nonmetro designations when examining rural/urban differences in health IT adoption, we also examined adoption across the OMB-defined countylevel dichotomous metro/nonmetro categories for comparative purposes. The results from bivariate analysis showed comparative rates between metropolitan and nonmetropolitan areas, providing stronger evidence that a rural-urban adoption gap is not significant in dentistry.

General practice clinics were found to be more likely to adopt a certification-capable EHR than specialty clinics. It has been suggested that a particular specialty has specific workflow and information needs that may differentiate them from other specialists and generalists. ${ }^{14}$ These needs may influence adoption decisions through an increase in uncertainty about the benefits from EHR systems. Sherer et $\mathrm{al}^{27}$ found that uncertain environments drive specialties to mimic and benchmark themselves against others within the same field, allowing organizations to hedge risks. It is expected then that trends in adoption may be present within varying specialty types. Larger practices, determined by the number of practicing dentists, were also found to be an important factor in predict- ing adoption. This has been a consistent finding in health IT adoption in both dental and medical clinics, highlighting the unique needs of smaller practices. ${ }^{12,19,52}$

We also found that the odds of adopting differed for two of the predictor variables between EDR and certification-capable EHR products. Dental clinics in medium rurality areas (0.345-0. 446 on IRR scale) were found to be less likely to adopt an EDR compared with clinics in urban areas (0.1330.236). Conversely, medium rurality clinics had a higher likelihood of adopting an EHR-capable system compared with urban clinics. Clinics in low and high rurality locations had comparable odds of adoption between models. General practice clinics were found to be significantly more likely to adopt an EHR-capable system than specialists, while the likelihood of adoption was similar between clinic types for EDRs. The odds of adoption in both models were found to be much greater in larger dental clinics ( $\geq 2$ dentists). However, this finding was significant in the EHR-defined model only. The differences in the odds of adoption between the two models for medium rurality areas and clinic type are difficult to interpret, as adoption was consistent for low and high rurality clinic locations and practice size. It is possible that specialty practices and medium rurality clinics may have certain inherent characteristics that constrain their ability to purchase certified systems as compared with basic EDRs. On the contrary, adoption differences could be due to the IT landscape itself, where certified systems are more accessible to generalists and dentists of certain locations. A more thorough understanding of these differences would necessitate an examination of adoption between varying dental specialities and across rural/urban definitions.

To begin the process of developing a truly integrated health IT ecosystem that includes dentistry as a critical component of one's overall health, there needs to be the development and use of certified EHRs in both the dental and medical fields. ${ }^{4}$ Data from this study indicate that dentistry is in a good position, but more work needs to be done. We believe that more studies examining EHR adoption in dentistry on a national scale are warranted, as state-level variation is a common finding in EHR adoption. ${ }^{53}$ Furthermore, there is a notable difference in adoption of a certification-capable EHR and a full EHR. Having a certification-capable system is more readily convertible to a full EHR than a system with no embedded upgradable EHR features. However, to comprehensively understand EHR adoption in dentistry in the United States, there needs to be an examination of the multifunctional capabilities $^{54,55}$ of adopted EHRs in all types of dental clinics. The benefits of legislating an increase in the use of certified EHRs in the dental field is unclear; however, policies to increase adoption that are mindful of potential disparities in IT use in smaller practices and between dental specialties and generalist may have special promise for success.

\section{Limitations}

The response rate for this study was relatively low at $36 \%$ indicating a $64 \%$ nonresponse bias, with nonrespondents more likely to be from more urban areas. Quantitative 
psychometric properties of survey instrument were not determined. Clustering techniques have the chance of increasing sampling error. The continuous measure for rurality used in the study (IRR) was categorized through a $\mathrm{K}$ means clustering technique. Categorizing this variable may have reduced the natural variation of rurality in the population sample. ${ }^{56}$ To partly address these problems, poststratification weights were made for the analysis using population characteristics, as estimated by the sample frame.

\section{Conclusion}

Findings from this study indicate moderate to high levels of IT adoption in dental clinics. We find that the percent adoption of some type of EDR among dental clinics in Tennessee in 2017 is comparable to 2014-level estimates of percent adoption of basic and certified EHRs in office-based physician practices in the same state. Specialization and practice size were significant predictors of EHR-capable system adoption. Efforts to increase dental IT adoption should be mindful of potential disparities between larger and smaller practices as well as between dental specialists and generalists.

\section{Clinical Relevance Statement}

Oral health information is an important component of an individual's overall health data. One of the essential first steps to an integrated medical-dental patient data environment is the development, adoption, and widespread use of EHRs in both the dental and medical fields. However, less attention has been paid to the adoption of IT systems in the dental field. Findings from analogous studies in the medical field have helped researchers, policy makers, clinicians, and vendors make more informed decisions on how to increase IT adoption and provide a more integrated patient data environment across medical specialties and domains. In this study, we find moderate to high levels of overall EDR adoption in dental clinics, with organizational structure, namely, specialization and practice size, as significant predictors of adoption of EHR-capable systems.

\section{Multiple Choice Questions}

1. When anticipating potential disparities in certified EHR adoption in dentistry, which of the following practice characteristics are most important to consider?

a. Regional location

b. Rural/urban differences

c. Practice size

d. Percent Medicare revenue

Correct Answer: The correct answer is option c. We find that practice size is a significant predictor of EHR-capable systems. The relative frequency of EHR adoption in clinics consisting of one practicing dentist was $52 \%$ compared with $70 \%$ for clinics with two or more dentists. Further, clinics with two or more practicing dentists were associated with a much greater likelihood of adopting an EHR- capable system than a clinic with one practicing dentist (adjusted odds ratio $=3.09, p=0.009$ ). This is a consistent finding across health care contexts and highlights the unique needs of smaller practices. The regional location of the clinic and rural/urban differences (A and B) were not associated with EHR adoption in our study. Although we did not specifically examine percent Medicare revenue (D) of a clinic as a potential factor for influencing dental IT adoption, it is unlikely that it would play a major role. Traditional Medicare does not cover most dental care. As a result, Medicare patient volume and revenue in most dental clinics is low, limiting qualifications for the federal incentive program and influence in modulating adoption decisions.

2. Which of the following most accurately identifies the difference between a basic electronic dental record (EDR) and a certified electronic health record (EHR) system?

a. EDRs meet criteria for Meaningful Use/Promoting Interoperability

b. Certified EHRs meet criteria for Meaningful Use/Promoting Interoperability

c. EDRs record patient demographics, computerized prescription order entry, and record clinical notes

d. Certified EHRs record patient demographics, computerized prescription order entry, and record clinical notes

Correct Answer: The most accurate answer is option b. Certified EHRs are systems that meet functional criteria for Meaningful Use/Promoting Interoperability attestation. Basic EDRs can be considered comparable in functionality to basic EMRs, where stored data are not readily exchanged between various IT systems and, thereby, cannot be used for Meaningful Use/Promoting Interoperability attestation. Due to the lack of consistency in functionality, clinical terminology, data standards, and controlled vocabularies between various EDR systems, choice (C) is not the most accurate option. Although option d is true in most cases, it does not most accurately highlight the difference between an EDR and a certified EHR system.

\section{Protection of Human and Animal Subjects}

Interviews were administered by the authors with data collection and analysis procedures approved by the Vanderbilt University's Institutional Review Board for the protection of human subjects.

\section{Conflict of Interest}

None.

\section{Acknowledgments}

We thank the faculty, staff, and graduate students of the Center for Medicine, Health, and Society at Vanderbilt University for their support and guidance in the design and implementation of this work. Furthermore, we would like to thank the faculty of the master's program in Vision Science and Investigative Ophthalmology at the 
University of Miami Miller School of Medicine and the laboratory of Dr. Sanjoy K. Bhattacharya for their feedback and support.

\section{References}

1 Oral health in America: a report of the Surgeon General. J Calif Dent Assoc 2000;28(09):685-695

2 McCluggage RW. A History of the American Dental Association; A Century of Health Service. Chicago, IL: American Dental Association; 1959

3 Powell V. Integration of Medical and Dental Care and Patient Data. New York, NY: Springer; 2012

4 Powell VJH, Din FM. Rationale and need to articulate medical and dental data. In: Powell V, Din FM, Acharya A, Torres-Urquidy MH, eds. Integration of Medical and Dental Care and Patient Data. London, UK: Springer London; 2012:1-24

5 Acharya A, Shimpi N, Mahnke A, Mathias R, Ye Z. Medical care providers' perspectives on dental information needs in electronic health records. J Am Dent Assoc 2017;148(05):328-337

6 Dimitropoulos L, Rizk S. A state-based approach to privacy and security for interoperable health information exchange. Health Aff (Millwood) 2009;28(02):428-434

7 Thyvalikakath TP, Monaco V, Thambuganipalle HB, Schleyer T. A usability evaluation of four commercial dental computer-based patient record systems. J Am Dent Assoc 2008;139(12):1632-1642

8 Brunt CS, Bowblis JR. Health IT adoption, productivity and quality in primary care. Appl Econ 2014;46(15):1716-271

9 DesRoches CM, Campbell EG, Rao SR, et al. Electronic health records in ambulatory care-a national survey of physicians. $\mathrm{N}$ Engl J Med 2008;359(01):50-60

10 Dranove D, Garthwaite C, Li B, Ody C. Investment subsidies and the adoption of electronic medical records in hospitals. J Health Econ 2015;44:309-319

11 Hsiao CJ, Jha AK, King J, Patel V, Furukawa MF, Mostashari F. Officebased physicians are responding to incentives and assistance by adopting and using electronic health records. Health Aff (Millwood) 2013;32(08):1470-1477

12 Singh R, Lichter MI, Danzo A, Taylor J, Rosenthal T. The adoption and use of health information technology in rural areas: results of a national survey. J Rural Health 2012;28(01):16-27

13 Xierali IM, Phillips RL Jr, Green LA, Bazemore AW, Puffer JC. Factors influencing family physician adoption of electronic health records (EHRs). J Am Board Fam Med 2013;26(04):388-393

14 Grinspan ZM, Banerjee S, Kaushal R, Kern LM. Physician specialty and variations in adoption of electronic health records. Appl Clin Inform 2013;4(02):225-240

15 Office-based Physician Health IT Adoption and Use; 2015. Available at: https://dashboard.healthit.gov/datadashboard/documen tation/physician-health-it-adoption-use-data-documentation. php. Accessed July 12, 2018

16 Abramovicz-Finkelsztain R, Barsottini CG, Marin HF. Electronic dental records system adoption. Stud Health Technol Inform 2015;216:17-20

17 Schleyer T, Song M, Gilbert GH, et al. Electronic dental record use and clinical information management patterns among practitioner-investigators in the Dental Practice-Based Research Network. J Am Dent Assoc 2013;144(01):49-58

18 Schleyer TK, Thyvalikakath TP, Spallek H, Torres-Urquidy MH, Hernandez P, Yuhaniak J. Clinical computing in general dentistry.J Am Med Inform Assoc 2006;13(03):344-352

19 Acharya A, Schroeder D, Schwei K, Chyou PH. Update on electronic dental record and clinical computing adoption among dental practices in the United States. Clin Med Res 2017;15(3-4):59-74

20 Ford DT. Are electronic health records the future of dental practice? J Calif Dent Assoc 2015;43(05):238-243
21 Health Professional Licensing Reports; 2017. Available at: https://apps.health.tn.gov/licensurereports/default.aspx. Accessed July 31, 2018

22 Schwei KM, Cooper R, Mahnke AN, Ye Z, Acharya A. Exploring dental providers' workflow in an electronic dental record environment. Appl Clin Inform 2016;7(02):516-533

23 Ward MM, Jaana M, Bahensky JA, Vartak S, Wakefield DS. Clinical information system availability and use in urban and rural hospitals. J Med Syst 2006;30(06):429-438

24 Dental Clinics; 2018. Available at: https://www.tn.gov/health/healthprogram-areas/oralhealth/clinics.html. Accessed July 12, 2018

25 Waldorf BS. A continuous multi-dimensional measure of rurality: moving beyond threshold measures. Annual Meeting of the American Agricultural Economics Association, Long Island, CA; 2006

26 Roehrich-Patrick L, Moreo B, Gibson T. Just How Rural or Urban are Tennessee's 95 Counties? Nashville, TN: Tennessee Advisory Commission on Intergovernmental Relations; 2016

27 Sherer SA, Meyerhoefer CD, Peng L. Applying institutional theory to the adoption of electronic health records in the U.S. Inf Manage 2016;53(05):570-801

28 Specialty Definitions; 2018. Available at: https://www.ada.org/ en/education-careers/careers-in-dentistry/dental-specialties/ specialty-definitions. Accessed July 12, 2018

29 Garrett P. EMR vs EHR-What is the Difference?; 2011 Available at: https://www.healthit.gov/buzz-blog/electronic-health-andmedical-records/emr-vs-ehr-difference/. Accessed July 12, 2018

30 EHR Products Used for Meaningful Use Attestation; 2017. Available at: https://dashboard.healthit.gov/datadashboard/documentation/ ehr-products-mu-attestation-data-documentation.php. Accessed July 20, 2018

31 Holmgren AJ, Adler-Milstein J, McCullough J. Are all certified EHRs created equal? Assessing the relationship between EHR vendor and hospital meaningful use performance. J Am Med Inform Assoc 2018;25(06):654-660

32 Torres-Urquidy MH, Powell VJH, Din FM, et al. HIT considerations: informatics and technology needs and considerations. In: Powell V, Din FM, Acharya A, Torres-Urquidy MH, eds. Integration of Medical and Dental Care and Patient Data. London, UK: Springer London; 2012:25-137

33 The Office of the National Coordinator for Health Information Technology (ONC). Connecting Health Care for the Nation. A shared nationwide Interoperability Roadmap; 2015. Available at: https://www.healthit.gov/sites/default/files/nationwide-interoperability-roadmap-draft-version-1.0.pdf. Accessed July 20, 2018

34 Certified Health IT Product List; 2015. Available at: https://chpl. healthit.gov/\#/search. Accessed July 20, 2018

35 Wilkin GA, Huang X. A practical comparison of two K-means clustering algorithms. BMC Bioinformatics 2008;9(Suppl 6):S19

36 Cochran WG. Some methods for strengthening the common $X^{2}$ tests. Biometrics 1954;10(04):417-511

37 Sherer S. Information Systems and Healthcare XXXIII: an institutional theory perspective on physician adoption of electronic health records. Comm Assoc Inform Syst 2010;26(01):71

38 Heisey-Grove DM. Variation in rural health information technology adoption and use. Health Aff (Millwood) 2016;35(02):365-370

39 Gold M, McLaughlin C. Assessing HITECH implementation and lessons: 5 years later. Milbank Q 2016;94(03):654-687

40 Office-based PEHRA. Health IT Quick-Stat \#50. Washington, DC: Office of the National Coordinator for Health Information Technology; 2016

41 Jaffe S. Seeking dental care for older Americans. Health Aff (Millwood) 2016;35(12):2164-2167

42 Boonstra A, Broekhuis M. Barriers to the acceptance of electronic medical records by physicians from systematic review to taxonomy and interventions. BMC Health Serv Res 2010;10:231

43 Jensen TB, Kjærgaard A, Svejvig P. Using institutional theory with sensemaking theory: a case study of information system implementation in healthcare. J Inf Technol 2009;24(04):343-531 
44 Mertz E. Reshaping professional boundaries and organizational forms in American dentistry: a case study of registered dental hygienists in alternative practice [dissertation]. San Francisco, CA: University of California; 2010

45 Currie W, Shoib G. Contextualising the IT artefact: towards a wider research agenda for IS using institutional theory. Inf Technol People 2009;22(01):63-771

46 Harris R, Brown S, Holt R, Perkins E. Do institutional logics predict interpretation of contract rules at the dental chair-side? Soc Sci Med 2014;122:81-89

47 O'Shea RM. Dentistry as an organization and institution. Milbank Mem Fund Q 1971;49(03):13-281

48 Kitchener M, Mertz E. Professional projects and institutional change in healthcare: the case of American dentistry. Soc Sci Med 2012;74(03):372-380

49 Swanson EB. Special issue on institutional theory in information systems research: contextualizing the IT artefact. J Inf Technol 2009;24(04):283-851
50 Kilaru AS, Wiebe DJ, Karp DN, Love J, Kallan MJ, Carr BG. Do hospital service areas and hospital referral regions define discrete health care populations? Med Care 2015;53(06):510-516

51 Wendling W. The private practice of dentistry. Oral Health Workforce in the Coming Decade: Workshop Summary, Washington, DC; 2009

52 Menachemi N, Langley A, Brooks RG. The use of information technologies among rural and urban physicians in Florida. J Med Syst 2007;31(06):483-488

53 Xierali IM, Hsiao CJ, Puffer JC, et al. The rise of electronic health record adoption among family physicians. Ann Fam Med 2013;11(01):14-19

54 Schoen C, Osborn R, Doty MM, Squires D, Peugh J, Applebaum S. A survey of primary care physicians in eleven countries, 2009: perspectives on care, costs, and experiences. Health Aff (Millwood) 2009;28(06):w1171-w1183

55 Huang MZ, Gibson CJ, Terry AL. Measuring electronic health record use in primary care: a scoping review. Appl Clin Inform 2018;9(01):15-33

56 Fowler FJ. Survey Research Methods. 4th ed. Thousand Oaks, CA: Sage Publications; 2009

\section{Appendix A Survey Overview}

\begin{tabular}{|c|c|}
\hline $\begin{array}{l}\text { 1. Does your practice currently use an electronic dental record } \\
\text { (EDR) keeping system of some kind? } \\
\square \text { Yes }^{*} \\
\square \text { No }^{\dagger} \\
\text { *If yes, what is the name of the EDR system you are using? }\end{array}$ & $\begin{array}{l}{ }^{\dagger} \text { If no, are you predominantly paper based? } \\
\square \text { Yes } \\
\square \text { No }\end{array}$ \\
\hline $\begin{array}{l}\text { 2. What type of practice is this? } \\
\square \text { Private } \\
\square \text { Group } \\
\square \text { Corporate } \\
{ }^{* \dagger} \text { If specialty or both, what type of specialty is practices? }\end{array}$ & $\begin{array}{l}\text { 3) Is this a specialty practice or a general practice? } \\
\qquad \text { General } \\
\square \text { Specialty* }^{*} \\
\square \text { Both }^{\dagger}\end{array}$ \\
\hline $\begin{array}{l}\text { 4) How many dentists work at this practice? } \\
\square \text { One } \\
\square \text { Two } \\
\square \text { (Three or more) } \\
\text { *Do you take Medicare or Medicaid patients? } \\
\square \text { Yes } \\
\square \text { No }\end{array}$ & $\begin{array}{l}\text { 5) Do you take insurance of any kind? } \\
\square \text { Yes* } \\
\square \text { No }\end{array}$ \\
\hline \multicolumn{2}{|l|}{ 6) Approximately how many patients do you see per month? } \\
\hline Additional Comments & \\
\hline
\end{tabular}

